\title{
Riñones poliquísticos: ¿cuándo retirarlos?, qué estudios requieren en seguimiento
}

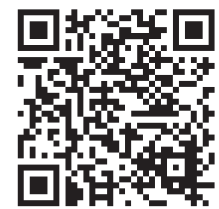

\author{
Polycystic kidneys: when is pre- post-transplant \\ nephrectomy indicated? Follow-up studies
}

\author{
Ramón Espinoza-Pérez* \\ * Jefe de la Unidad de Trasplantes. Hospital de Especialidades. Centro Médico Nacional Siglo XXI. Instituto Mexicano del Seguro Social.
}

\section{INTRODUCCIÓN}

La enfermedad renal poliquística es la enfermedad renal hereditaria más frecuente. Se caracteriza por el desarrollo progresivo de múltiples quistes en los riñones que destruyen el parénquima renal. Cerca de $10 \%$ de los pacientes con insuficiencia renal terminal padecen esta enfermedad. Macroscópicamente se caracteriza por el aumento de tamaño de los riñones y por los quistes con gran cantidad de líquido seroso que forman la superficie.

Existen dos tipos de enfermedad renal poliquística: autosómica dominante y autosómica recesiva.

Puede haber manifestaciones extrarrenales de la enfermedad como aneurisma cerebral en $8 \%$, alteraciones cardiovasculares como prolapso de la válvula mitral, insuficiencia mitral y tricuspídea, divertículos en colon y quistes en otros órganos como hígado y páncreas.

1. Cuando los riñones poliquísticos son de gran tamaño o son quistes complicados se sugiere retirarlos antes del trasplante.

1.1. Si los quistes se encuentran complicados es deseable la nefrectomía como la mejor opción para retirarlos.

1.2. Si el riñón no tiene quistes complicados sugerimos nefrectomía o embolización para el retiro del mismo.
1.3. Es deseable llevar un seguimiento del riñón poliquístico que no se retira en el paciente trasplantado con ultrasonido y/o tomografía, y si hay cambios en los quistes (engrosamiento de pared, múltiples septos irregulares, calcificaciones, aumento en las UH o captación del medio de contraste) o si el paciente tiene síntomas se sugiere retirarlo por la posibilidad de absceso o malignización.

\section{LECTURAS RECOMENDADAS}

1. Drognitz O, Kirste G, Schramm I et al. Kidney transplantation with concomitant unilateral nephrectomy: a matched-pair analysis on complications and outcome. Transplantation. 2006; 81 (6): 874-880.

2. Perrone RD, Ruthazar R, Terrier NL. Survival after end-stage renal disease in autosomal dominant polycystic Kidney Disease contribution of external complications to mortality. Am J Kidney Dis. 2001; 38: 774-784.

3. Vega J, Lira D, Medel S, Betancour P, Goecke H, Carrasco A. Outcome of renal transplantation in patients with autosomal dominant polycystic kidney disease. Rev Med Chil. 2012; 140 (8): 990-998.

4. Rochefort A, Chacón R, Durruty J. Embolización de riñones poliquísticos una alternativa menos invasiva a la nefrectomía pretrasplante. Rev Chilena de Urología. 2015; 80 (2): 75-79.

5. Campbell Tratado de Urología. Enfermedad renal quística y displasia renal. Cap. 39, Tomo II. 8a. ed. Buenos Aires: Edit. Panamericana; 2004.

6. Pascual J, Abramowicz D, Cochat P, Claas F, Dudley C, Harden $\mathrm{P}$ et al. European renal best practice guideline on the management and evaluation of the kidney donor and recipient. Nefrologia. 2014; 34 (3): 293-301. 
7. Abramowicz D, Cochat P, Claas FH, Heemann U, Pascual J, Dudley $\mathrm{C}$ et al. European Renal Best Practice Guideline on kidney donor and recipient evaluation and perioperative care. Nephrol Dial Transplant. 2015; 30 (11): 1790-17897.
Correspondencia:

Dr. Ramón Espinoza-Pérez

E-mail: ramon.espinoza@imss.gob.mx

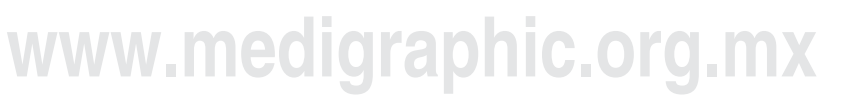

